Revista Calidad en la Educación Superior

Programa de Autoevaluación Académica

Universidad Estatal a Distancia

ISSN 1659-4703

Costa Rica

revistacalidad@uned.ac.cr

\title{
SISTEMATIZACIÓN DE LAS FORTALEZAS Y DEBILIDADES DE UN PLAN DE ESTUDIOS EN EL PROCESO ACREDITACIÓN-REACREDITACIÓN
}

\section{STRENGTHS AND WEAKNESSES OF A CURRICULUM IN AN ACCREDITATION AND RE-ACCREDITATION PROCESS}

\author{
Elizabeth González Sandoval ${ }^{1}$, Elizabeth.gonzalez.sandoval@una.cr \\ María Adilia García Vargas ${ }^{2}$, maría.garcía.vargas@una.cr \\ Esteban González Sandoval ${ }^{3}$,esteban.gonzalez.sandoval@una.cr \\ Universidad Nacional de Costa Rica, Costa Rica
}

\author{
Volumen 5, Número 1 \\ Mayo 2014 \\ pp. $119-135$
}

Recibido: enero, 2014

Aprobado: abril, 2014

\footnotetext{
${ }^{1}$ Universidad Nacional de Costa Rica, Escuela de Informática, elizabeth.gonzalez.sandoval@una.cr

2 Universidad Nacional de Costa Rica, Escuela de Informática, maria.garcia.vargas@una.cr

3 Universidad Nacional de Costa Rica, Escuela de Bibliotecología, esteban.gonzalez.sandoval@una.cr
} 
Sistematización de las fortalezas y debilidades de un plan de estudios en el proceso acreditación-reacreaditación

Elizabeth González Sandoval, María Adilia García Vargas, Esteban González Sandoval

\section{Resumen}

El plan de Estudios de la carrera Ingeniería en Sistemas de Información con grado de Bachillerato de la Escuela de Informática de la Universidad Nacional, fue acreditado siguiendo las especificaciones dadas por el Sistema Nacional de Acreditación de la Educación Superior (SINAES) a partir del 11 de agosto del año 2005, 4 años después de esta acreditación correspondía iniciar el proceso de reacreditación, por lo que se lleva a cabo un análisis de las debilidades y fortalezas del Plan de Estudio, con el fin de proponer acciones que se deberán realizar para mejorar la calidad de dicho plan, los resultados se presentaron en el Informe de Ejecutoría (2005) y se basó en los 6 componentes que conformaban el modelo de acreditación utilizado en el año 2005 a saber: Personal Académico, Currículum, Estudiantes, Administración, Estructura y Equipamiento e Impacto y Pertinencia. Para el año 2008 el SINAES crea un nuevo modelo que deberá utilizarse para los siguientes procesos de autoevaluación.

El análisis de cada componente es bastante extenso por lo tanto, en este artículo se presenta los resultados del primer componente: Personal Académico, posteriormente en otros artículos se presentarán los otros cinco componentes.

Se pretende compartir esta experiencia con aquellas instituciones que tienen intenciones de someterse a un proceso de autoevaluación, ya sea con fines de acreditación o solamente para mejorar la calidad de su Plan de Estudio.

Palabras clave: acreditación, reacreditación, modelo, autoevaluación, componente, Plan de Estudios, Informe de Ejecutoría.

\section{Abstract}

The curriculum of career in Information Systems Engineering with Bachelor's degree from the School of Computing, National University was accredited following the specifications given by the National Higher Education Accreditation (SINAES) from 11 August 2005, four years after starting this accreditation corresponded reaccreditation process, so it is carried out an analysis of the strengths and weaknesses Curriculum, to propose actions to be carried out to improve quality of the plan, the results are presented in the report execution (2005) and was based on the six components that comprise the accreditation model used in 2005 are: Academic Personnel, Curriculum, Student Administration, Structure and Equipment and Impact and Relevance. For 2008 the SINAES creates a new model to be used for the following self-assessment processes.

The analysis of each component is quite extensive so, this article presents the results of the first two components Curriculum Faculty and later in another article will present the 
Student and Administration component and a third article in the latest components namely infrastructure and Equipment and Impact and Relevance.

It aims to share this experience with those institutions who intend to undergo a selfassessment process, either for accreditation or just to improve the quality of its curriculum.

Keywords: accreditation, reaccreditation, model, self-assessment component, Curriculum, report execution.

\section{Introducción}

Como se indicó en el Informe de Autoevaluación para la Reacreditación (2009) "La Escuela de Informática está comprometida en un esfuerzo de mejoramiento continuo en su quehacer académico y administrativo que le permita responder, en su área de especialidad, de manera efectiva y contundente a las expectativas y necesidades de la sociedad costarricense."

El proceso de autoevaluación para la reacreditación debió considerar tanto una valoración de los avances logrados al implementar el Plan de Mejoramiento (2005), así como una nueva autoevaluación que permitiera diagnosticar el estado actual de la carrera y de la Escuela, y establecer nuevas metas a lograr. En este proceso participaron profesionales tanto externos como internos, se aplicaron instrumentos, se realizaron reuniones y talleres con estudiantes, académicos, administrativos, graduados y empleadores; para conocer las fortalezas y debilidades del plan de estudio, su integración, soporte logístico y recurso humano.

Este proceso ha sido guiado por las pautas y especificaciones sugeridas por el SINAES, es el producto de un trabajo comprometido de toda la Escuela de Informática que fructificará graduandos profesionales con calidad, no sólo con capacidades en nuestras áreas de saber, sino en su desarrollo humano integral. 
Como resultado del Proceso de Autoevaluación, se elaboró una comparación entre las fortalezas y debilidades o necesidades identificadas en el primer proceso de autoevaluación del año 2005 y las que se identificaron en el segundo proceso de autoevaluación realizado en el año 2009, es decir cuatro años después. Además, para completar la información, se realizó una valoración analizando estos resultados para ofrecer el punto de vista y los compromisos de la Escuela, ante estos resultados. Siempre dentro del ámbito permitido por la diferencia entre el modelo utilizado en el año 2005 y el modelo utilizado en el año 2009.

\section{Componente: Personal Académico}

Este componente comprende todos los aspectos relacionados con el personal académicos involucrados en la ejecución del Plan de estudio que está siendo autoevaluado. Este componente está formado por 6 subcomponentes, en cada uno de ellos se describe las fortalezas y debilidades que llevan a establecer acciones que se deben ejecutar con miras al mejoramiento del desarrollo del Plan de Estudios.

\section{a. Estabilidad del personal docente}

Basados en los estándares del modelo del SINAES y los resultados de los instrumentos aplicados junto con los datos recopilados de las reuniones y talleres realizados con los académicos, se determinan las siguientes Fortalezas y Debilidad. Se propone para éstas acciones de mejoramiento que permitirán solventar las debilidades. 


\section{Cuadro 1}

Fortalezas, debilidades y acciones de mejoramiento de personal docente

\begin{tabular}{|c|c|c|}
\hline Fortalezas & Debilidades & Acción de mejoramiento \\
\hline $\begin{array}{l}\text { 1. El } 100 \% \text { de los académicos } \\
\text { propietarios tienen más de } \\
\text { cinco años de laborar para la } \\
\text { carrera y sobre los } \\
\text { académicos interinos el } \\
59.26 \% \text { tiene más de tres } \\
\text { años de laborar para la } \\
\text { carrera. } \\
\text { 2. De los } 26 \text { cursos del área } \\
\text { informática contemplados en } \\
\text { el plan de estudios de la } \\
\text { carrera, para el período } 2000 \\
\text { al } 2004 \text {, únicamente el } 31 \% \text { ha } \\
\text { cambiado totalmente su } \\
\text { nómina de profesores una vez, } \\
\text { lo cual muestra una gran } \\
\text { estabilidad del personal } \\
\text { docente que imparte los } \\
\text { cursos. }\end{array}$ & $\begin{array}{l}\text { 1. La Escuela debe contar con } \\
\text { un mayor número de } \\
\text { profesores contratados en } \\
\text { propiedad para potenciar una } \\
\text { mayor estabilidad del cuerpo } \\
\text { docente. Se requiere llegar a } \\
\text { una relación mínima de } 70 \% \\
\text { propietarios y } 30 \% \text { interinos. }\end{array}$ & $\begin{array}{l}\text { La Escuela gestionará ante las } \\
\text { Autoridades Universitarias la } \\
\text { conversión de al menos seis } \\
\text { tiempos completos de } \\
\text { interinos en propietarios, para } \\
\text { potenciar una mayor } \\
\text { estabilidad del cuerpo } \\
\text { docenter }\end{array}$ \\
\hline $\begin{array}{l}\text { 3. La Escuela cuenta con un } \\
\text { adecuado número de } \\
\text { académicos por área de } \\
\text { especialidad, lo cual garantiza } \\
\text { que los diversos cursos del } \\
\text { plan de estudios de la carrera } \\
\text { se puedan impartir en los } \\
\text { ciclos respectivos. }\end{array}$ & & \\
\hline $\begin{array}{l}\text { 4. La cantidad de personal } \\
\text { docente de la Escuela de } \\
\text { Informática durante los últimos } \\
\text { cuatro años ha permitido } \\
\text { atender adecuadamente la } \\
\text { demanda de la oferta } \\
\text { académica de los estudiantes } \\
\text { estipulada en el plan de }\end{array}$ & & \\
\hline
\end{tabular}

Revista CAES Vol. 5, No. 1, Año 2014 ISSN-1659-4703 


\begin{tabular}{|l|l|l|}
\hline estudio de la carrera, & & \\
permitiendo incluso ofrecer & & \\
cursos en ciclos lectivos que & & \\
no corresponden, como & & \\
respuesta a la demanda de los & & \\
estudiantes. & & \\
\hline $\begin{array}{l}\text { 5. La Escuela cuenta con un } \\
\text { personal académico } \\
\text { relativamente joven, } \\
\text { laboralmente hablando, lo que }\end{array}$ & & \\
garantiza que al corto o & & \\
mediano plazo no se tendrán & & \\
problemas de jubilación. & & \\
\hline
\end{tabular}

Fuente: elaboración propia, 2014

\section{b. Jornadas e interacción docentes - estudiantes}

Según el análisis de las jornadas académicas asignadas a la carrera se detecta las siguientes fortalezas y debilidades, también se proponen acciones de mejoramiento para solventar las debilidades encontradas.

\section{Cuadro 2}

Fortalezas, debilidades y acciones de mejoramiento de jornadas académicas

\begin{tabular}{|l|l|l|}
\hline \multicolumn{1}{|c|}{ Fortalezas } & \multicolumn{1}{|c|}{ Debilidades } & Acción de mejoramiento \\
\hline 1. En promedio el $50 \%$ de las & 1. La opinión del 51.1\% de & 1. Desarrollar un plan para \\
jornadas docentes asignadas a la & los estudiantes consideran & divulgar, incentivar y motivar a \\
carrera son iguales o superiores & que la disponibilidad y y & los estudiantes a utilizar las \\
al medio tiempo completo, con lo & accesibilidad de los docentes & horas de consulta extra-clase \\
que se logra disponer de un & extra-clase no es la & ofrecidas por los docentes y \\
cuerpo de profesores de planta, & adecuada, de igual forma un & garantizar la disponibilidad del \\
con presencia permanente en la & $84.4 \%$ de los docentes & cuerpo docente en este \\
Unidad. & encuestados indican que el & periodo. \\
& $\begin{array}{ll}\text { en o menos de los } \\
\text { estudiantes utilizan las horas }\end{array}$ \\
\hline
\end{tabular}




\begin{tabular}{|c|c|}
\hline & extra-clase. \\
\hline $\begin{array}{l}\text { 2. La presencia de este cuerpo } \\
\text { docente brinda la posibilidad de } \\
\text { espacios de reflexión y análisis, } \\
\text { que el desarrollo y gestión que la } \\
\text { Unidad Académica requiere. } \\
\text { Además posibilita que los } \\
\text { docentes asesoren y guíen a los } \\
\text { estudiantes, en aspectos } \\
\text { curriculares, en promedio el } 77 \% \\
\text { de ellos así lo indican en la } \\
\text { encuesta realizada a éstos. }\end{array}$ & \\
\hline $\begin{array}{l}\text { 3. Las horas de atención a } \\
\text { estudiantes, extra-clase, están } \\
\text { debidamente establecidas y } \\
\text { reconocidas como parte de la } \\
\text { carga docente y son definidas en } \\
\text { común acuerdo con los } \\
\text { estudiantes. }\end{array}$ & \\
\hline $\begin{array}{l}\text { 4. La percepción de los } \\
\text { estudiantes en un } 82 \% \text { considera } \\
\text { que la disponibilidad y } \\
\text { accesibilidad de los docentes } \\
\text { durante la clase es más que } \\
\text { adecuada. }\end{array}$ & \\
\hline
\end{tabular}

Fuente: elaboración propia, 2014

\section{c. Tiempo para participar en actividades de investigación y desarrollo profesional}

Según la normativa institucional sobre la carga docente y la participación académica en actividades de investigación y actualización se detectan las siguientes fortalezas y debilidades. Se proponen acciones de mejoramiento para solventar las debilidades encontradas. 


\section{Cuadro 3 \\ Fortalezas, debilidades y acciones de mejoramiento de carga docente y la participación académica en actividades de investigación y actualización}

\begin{tabular}{|c|c|c|}
\hline Fortalezas & Debilidades & Acción de mejoramiento \\
\hline $\begin{array}{l}\text { 1. La Universidad Nacional } \\
\text { provee un marco normativo y } \\
\text { filosófico que permite y } \\
\text { propicia la participación de sus } \\
\text { académicos en actividades de } \\
\text { investigación y desarrollo } \\
\text { profesional. }\end{array}$ & $\begin{array}{l}\text { 1. Las jornadas académicas de la } \\
\text { Unidad se han utilizado } \\
\text { prioritariamente en la docencia. La } \\
\text { extensión como función académica } \\
\text { se ha asumido por recargo o bien } \\
\text { incorporándola como parte del } \\
\text { quehacer docente en los cursos de } \\
\text { carácter práctico. }\end{array}$ & $\begin{array}{l}\text { 1. La Escuela gestionará ante } \\
\text { las Autoridades Universitarias } \\
\text { la asignación de un tiempo } \\
\text { completo académico por año, } \\
\text { durante los próximos tres } \\
\text { años, el cual será utilizado } \\
\text { para potenciar el desarrollo de } \\
\text { la extensión e investigación en } \\
\text { la Unidad. De igual forma, } \\
\text { desarrollará un plan para } \\
\text { incentivar y motivar a su } \\
\text { personal académico a realizar } \\
\text { proyectos en estas áreas del } \\
\text { quehacer universitario. }\end{array}$ \\
\hline $\begin{array}{l}\text { 2. La Unidad Académica } \\
\text { reconoce la importancia de la } \\
\text { realización de actividades de } \\
\text { investigación y desarrollo } \\
\text { profesional y su incidencia en } \\
\text { la calidad de la carrera. }\end{array}$ & $\begin{array}{l}\text { 2. La investigación como pilar fundar } \\
\text { del quehacer } \\
\text { docente debe ser potenciada con la } \\
\text { asignación efectiva de } \\
\text { recursos tanto laborales como de } \\
\text { operación }\end{array}$ & \\
\hline $\begin{array}{l}\text { 3. La Escuela de } \\
\text { Informática ha propiciado } \\
\text { en los últimos cuatro } \\
\text { años investigaciones } \\
\text { en el área; asignando } \\
\text { carga académica a } \\
\text { los profesores que } \\
\text { formulan y presentan } \\
\text { proyectos }\end{array}$ & & \\
\hline $\begin{array}{l}\text { 4. Actualmente se está } \\
\text { desarrollando un plan } \\
\text { estratégico para definir las }\end{array}$ & & \\
\hline
\end{tabular}

Revista CAES Vol. 5, No. 1, Año 2014 ISSN-1659-4703 


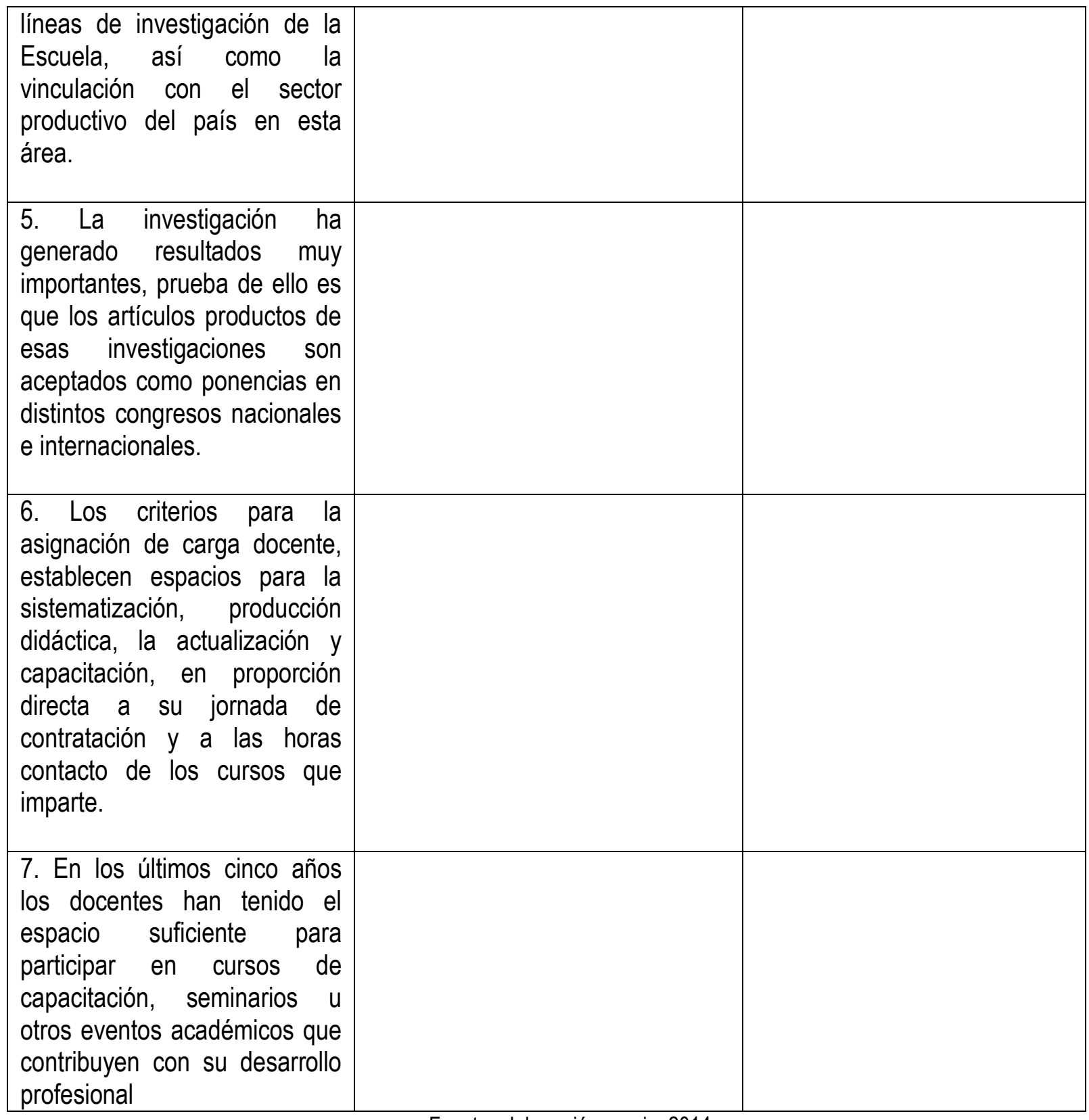




\section{d. Liderazgo y reconocimiento del Director}

Según normativa institucional y el perfil del director en turno se detectan las siguientes fortalezas.

\section{Cuadro 4. Liderazgo y reconocimiento del Director}

\begin{tabular}{|c|c|c|}
\hline Fortalezas & Debilidades & Acción de mejoramiento \\
\hline $\begin{array}{l}\text { 1. El sistema de elección de director de la } \\
\text { Unidad Académica no es restrictivo y } \\
\text { permite que democráticamente la } \\
\text { Asamblea de Escuela, elija la persona } \\
\text { que liderará la Escuela por cinco años. }\end{array}$ & & \\
\hline $\begin{array}{l}\text { 2. El actual director de la Escuela cuenta } \\
\text { con demostrada solvencia académica } \\
\text { profesional, reconocido a nivel } \\
\text { nacional e internacional. } \\
\text { Su conocimiento de la institución y } \\
\text { experiencia docente y profesional ha } \\
\text { sido clave en el desarrollo y proyección } \\
\text { de esta Unidad. Actualmente es el } \\
\text { representante por Costa Rica de la Red } \\
\text { lbero-americana de Informática } \\
\text { Educativa (RIBIE). }\end{array}$ & & \\
\hline
\end{tabular}

\section{e. Nivel de competencia de los docentes}

Según el análisis y el perfil curricular y la experiencia de cada docente se han detectado loas siguientes fortalezas y debilidades. Se propone acciones de mejoramiento para solventar las debilidades detectadas. 


\section{Cuadro 5. Nivel de competencia de los docentes}

\begin{tabular}{|c|c|c|}
\hline $\begin{array}{r}\text { Fortalezas } \\
\end{array}$ & Debilidades & Acción de mejoramiento \\
\hline $\begin{array}{l}\text { 1. En promedio el } 60 \% \text { de los } \\
\text { docentes que han atendido la } \\
\text { oferta académica de la carrera } \\
\text { durante los últimos cinco años, } \\
\text { poseen grados académicos de } \\
\text { licenciatura, maestría o } \\
\text { doctorado, más del } 25 \% \text { tiene } \\
\text { grado académico de pos- } \\
\text { grado, lo que asegura un buen } \\
\text { nivel de competencia } \\
\text { académica. El } 82.3 \% \text { de los } \\
\text { estudiantes opinan que el nivel } \\
\text { académico de los profesores } \\
\text { es adecuado o muy adecuado. }\end{array}$ & $\begin{array}{l}\text { 1. Existe la necesidad de que } \\
\text { los profesores con grado de } \\
\text { Bachillerato alcancen un } \\
\text { grado académico mayor. }\end{array}$ & $\begin{array}{l}\text { La Unidad Académica } \\
\text { garantizará la ejecución del } \\
\text { plan de capacitación dirigido a } \\
\text { los profesores con grado de } \\
\text { bachillerato, para que en un } \\
\text { plazo no mayor a tres años } \\
\text { todo académico de la Escuela } \\
\text { cuente con el grado mínimo de } \\
\text { licenciatura. }\end{array}$ \\
\hline $\begin{array}{l}\text { 2. En promedio un } 60 \% \text { de los } \\
\text { docentes ha estado nombrado } \\
\text { en categoría del régimen } \\
\text { académico de instructor } \\
\text { licenciado o superior. }\end{array}$ & $\begin{array}{l}\text { Existe la necesidad de que los } \\
\text { profesores con grado de } \\
\text { Bachillerato alcancen un } \\
\text { grado académico mayor. }\end{array}$ & \\
\hline $\begin{array}{l}\text { 3. Las áreas de formación y } \\
\text { especialización de los } \\
\text { docentes guardan estrecha } \\
\text { relación con el plan de } \\
\text { estudios y los contenidos de } \\
\text { los cursos. }\end{array}$ & & \\
\hline $\begin{array}{l}\text { 4. El } 75 \% \text { de los académicos } \\
\text { en propiedad tienen más de } \\
10 \text { años de experiencia } \\
\text { docente y el } 100 \% \text { cuenta } \\
\text { con una experiencia docente } \\
\text { de más de } 5 \text { años. Con } \\
\text { respecto a los profesores } \\
\text { interinos el } 50 \% \text { posee una } \\
\text { experiencia docente mayor o } \\
\text { igual a } 3 \text { años. }\end{array}$ & & \\
\hline
\end{tabular}

Revista CAES Vol. 5, No. 1, Año 2014 ISSN-1659-4703 


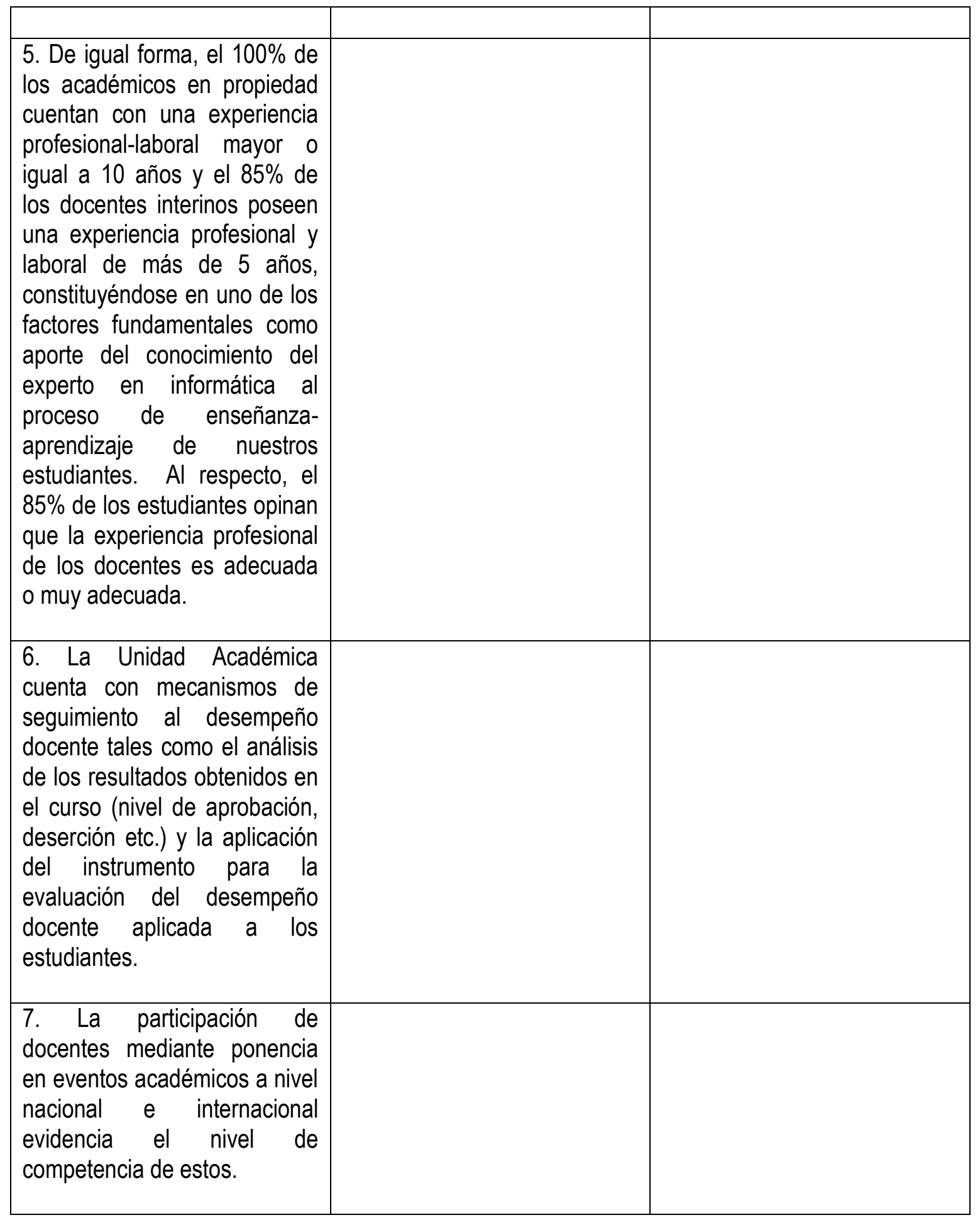




\section{f. Capacitación, perfeccionamiento y actualización}

La Universidad Nacional ofrece opciones de capacitación a nivel institucional que permiten que el académico se actualice o perfeccione en algún área del saber. Un análisis del perfil del académico permitió que se detectaran las siguientes fortalezas.

\section{Cuadro 6. Capacitación, perfeccionamiento y actualización}

\begin{tabular}{|c|c|c|}
\hline Fortalezas & Debilidades & Acción de mejoramiento \\
\hline $\begin{array}{l}\text { 1. La institución brinda oportunidades } \\
\text { para la participación en cursos o } \\
\text { espacios de capacitación y } \\
\text { actualización y cuenta con recursos } \\
\text { institucionales y mecanismos para que } \\
\text { los funcionarios puedan cursar } \\
\text { estudios de posgrado. }\end{array}$ & & \\
\hline $\begin{array}{l}\text { 2. La Unidad Académica impulsa una } \\
\text { serie de estrategias y mecanismos } \\
\text { para mejorar el desempeño didáctico y } \\
\text { profesional de los docentes y los } \\
\text { resultados de la evaluación del } \\
\text { desempeño docente asílo confirman. }\end{array}$ & & \\
\hline $\begin{array}{l}\text { 3. La Escuela cuenta con recursos } \\
\text { económicos propios y ha sido política } \\
\text { del Consejo Académico de la Unidad } \\
\text { apoyar la participación y actualización } \\
\text { de los docentes en igualdad de } \\
\text { oportunidades en las diferentes áreas } \\
\text { de especialización. }\end{array}$ & & \\
\hline
\end{tabular}

Fuente: elaboración propia, 2014 


\section{Lecciones aprendidas}

La acreditación es un proceso necesario que permite que una institución educativa conozca cuales tareas se están haciendo bien, cuáles se están ejecutando incorrectamente o del todo no se están llevando a cabo. Se innovan procedimientos, se atienden situaciones y se valoran los resultados. A partir de ahí la Escuela de Informática se debe comprometer, por medio de mecanismos, a una mayor calidad docente.

Además, este proceso llevó a mejorar, en la Escuela de Informática y en la institución, los recursos de información disponibles para la gestión de la Escuela, por lo que se tuvo que sistematizar los datos y se generó una gran cantidad de información que permitió conocer el estado de la Escuela de Informática y al mismo tiempo la Universidad consolidó procedimientos que brindaran este tipo de información a otras carreras que se encontraban en el proceso de autoevaluación.

En estos cuatro años, la experiencia acumulada de la evaluación permanente de la carrera, ha permeado en los funcionarios, dando sentido al cumplimiento de las responsabilidades y obligaciones académicas ajustadas a los distintos parámetros de calidad, se puede decir que se está desarrollando realmente una cultura de calidad.

A pesar de los grandes esfuerzos que implica el proceso de acreditación, el trabajo participativo llevado a cabo contribuyó en una distribución más efectiva de las labores, y ha propiciado las buenas relaciones humanas en los(as) compañeros(as).

Los miembros de la Comisión de Acreditación de la carrera, encontraron a lo interno de la comisión una oportunidad para conocer a fondo el quehacer de la Escuela, las generalidades y especificidades de la Universidad, los procesos de evaluación, 
autoevaluación, mejoramiento, calidad, planificación, adaptación al nuevo modelo de SINAES, y además, se logró un aprendizaje significativo, lo que se puede notar en su producción académica. Los resultados del trabajo de la comisión demuestran que es un equipo consolidado y comprometido con el proceso, liderado por la dirección de la Escuela de Informática.

La recolección, análisis y elaboración de los informes de autoevaluación y acreditación son buenas experiencias que fortalecen la cultura y el aprendizaje sobre la investigación.

Los estudiantes fundamentados en la evaluación y criterios de calidad con los que debe contar la carrera, solicitan con más derecho mejoras y cambios en cualquier asunto que les afecte, existe una participación estudiantil abierta, consideramos que estos valores se mantendrán en la conciencia de ellos cuando como futuros profesionales sean exigentes en los puestos que desempeñen y por tanto, mejores ciudadanos aportando al desarrollo nacional.

La acreditación exige un compromiso muy fuerte por parte de la Escuela en optimizar los servicios, en vencer las debilidades y mantener las fortalezas, con el fin de satisfacer a los participantes involucrados de su comunidad y responder las demandas de los ciudadanos costarricenses.

Se aprovechan al máximo los recursos disponibles, planificando y ejecutando acciones pertinentes, evaluadas por los participantes. La adquisición y actualización de equipos ha sido un interés permanente en la Escuela. La dirección ha mantenido la estabilidad de los cursos que corresponde a cada profesor, con el fin de ir formando cátedras y especializaciones. 
Por tanto, se considera que, sin lugar a dudas, ha sido un éxito el proceso de acreditación en la Escuela de Informática ante las evidencias presentadas y los logros obtenidos, documentados en el informe de reacreditación y en estos dos componentes Personal Académico y Currículum del III informe de Ejecutoria del Plan de Mejoramiento (2009). Tal como se indicó anteriormente los cinco componentes que faltan se presentarán en artículos posteriores a éste.

\section{Referencias Bibliográficas}

Coto, M., Chávez, L., González, E., Mora, S., Segura, A. (2005). Informe Autoevaluación Escuela de Informática. UNA.

Coto, M., Chávez, L., González, E., Mora, S., Segura, A. (2005). Plan de Mejoramiento Escuela de Informática. UNA.

Coto, M., Chávez, L., González, E., Mora, S., Segura, A. (2005). Plan de Mejoramiento Escuela de Informática.

García, M., Lizano, F., González, E., Vílchez, E., Sánchez, J., Sandoval, M., Trujillo, A. (2009). Compromiso de Mejoramiento Escuela de Informática 2009.

García, M., Lizano, F., González, E., Vílchez, E., Sánchez, J., Sandoval, M., Trujillo, A. (2009). Informe Autoevaluación. Escuela de Informática.

Ley 8798 "Fortalecimiento del Sistema Nacional de Acreditación de la Educación Superior (SINAES)", 2010. 
Sistematización de las fortalezas y debilidades de un plan de estudios en el proceso acreditación-reacreaditación

Elizabeth González Sandoval, María Adilia García Vargas, Esteban González Sandoval

SINAES. (2003). Modelo de autoevaluación. Recuperado de http://www.sinaes.ac.cr

SINAES. (2009). Manual de acreditación oficial de carreras de grado del Sistema Nacional de Acreditación de la Educación Superior. San José, Costa Rica:

SINAES. (2009). Manual de acreditación oficial de carreras de grado del Sistema Nacional de Acreditación de la Educación Superior. San José, Costa Rica: SINAES. 